\title{
Experiencia del aula invertida para promover estudiantes prosumidores del nivel superior
}

\section{(A flipped classroom experience to promote prosumer students of the higher level)}

\author{
María Obdulia González Fernández \\ Pablo Huerta Gaytán \\ Universidad de Guadalajara, UdeG (México)
}

DOI: http://dx.doi.org/10.5944/ried.22.2.23065

\section{Cómo referenciar este artículo:}

González Fernández, M. O., y Huerta Gaytán, P. (2019). Experiencia del aula invertida para promover estudiantes prosumidores del nivel superior. RIED. Revista Iberoamericana de Educación a Distancia, 22(2), pp. 245-263. doi: http://dx.doi.org/10.5944/ried.22.2.23065

\section{Resumen}

Este trabajo describe una experiencia académica del aula invertida implementada como estrategia en la asignatura de Promoción, impartida a estudiantes del segundo semestre de Ingeniería en Computación, de la Universidad de Guadalajara. El propósito principal fue promover el consumo y producción de recursos educativos. Se planeó la aplicación de una estrategia didáctica dividida en tres momentos: aula invertida con recursos propuestos por el profesor de acuerdo con el estilo de aprendizaje del estudiante; la creación de recursos didácticos por parte del alumnado; y finalmente, la evaluación a partir de un estudio descriptivo a una muestra de 24 sujetos. Durante el estudio se aplicó inicialmente un cuestionario tipo Likert organizado en torno a la percepción metodológica referida al aprendizaje, a la interacción docente-estudiante, estudiante-estudiante, ritmos de aprendizaje, colaboración, resolución de problemas, motivación y actitud prosumidora. Los resultados destacan que el 88\% están de acuerdo en la metodología y personalización de ritmos de aprendizaje; un 92\% afirma que la estrategia permitió mejorar su aprendizaje y habilidades de comunicación; por lo anterior se deduce una mayor interacción entre ellos y el profesor, lo que desarrolló su pensamiento crítico y habilidades para resolver problemas; se pudieron integrar estrategias para la creación y difusión de contenidos; se aumentó la motivación del alumno para colaborar y comprobar el principio constructivista de aprender haciendo. La aplicación de modelos didácticos activos requiere la conformación de ambientes de aprendizajes bajo principios del estilo del aprendiz e integrar sus intereses para el desarrollo de sus competencias. 
Palabras clave: aula invertida; modelo didáctico; ambiente de aprendizaje; método activo; tecnologías de la información y de la comunicación; estudiantes.

\begin{abstract}
This work describes an academic experience of the flipped classroom implemented as a strategy in the subject of Promotion, given to students of the second semester of Computer Engineering, of the University of Guadalajara. The main purpose was to promote the consumption and production of educational resources. The application of a didactic strategy divided into three moments was planned: classroom inverted with resources proposed by the teacher, based on the student's learning styles; participation of students in the creation of teaching resources; and finally, the evaluation from a descriptive study to a sample of 24 subjects. During the study, a Likert-type questionnaire was initially applied, organized around the methodological perception related to learning, the teacher-student interaction, studentstudent, learning rhythms, collaboration, problem solving, motivation and prosuming attitude. The results highlight that $88 \%$ agree on the methodology and personalization of learning rhythms; $92 \%$ state that the strategy allowed them to improve their learning and communication skills; for the previous thing one deduces a greater interaction between them and the professor, which developed his critical thought and abilities to solve problems; strategies for creating and disseminating content could be integrated; the student's motivation to collaborate and check the constructivist principle of learning by doing was increased. The application of active didactic models requires the conformation of learning environments under the principles of the learner's style and integrating their interests for the development of their competences.
\end{abstract}

Keywords: inverted classroom; didactic model; learning environment; active method; information and communication technologies; students.

Actualmente el modelo tradicional de clase magistral no cumple con todas las expectativas para que los estudiantes obtengan los resultados esperados en el desarrollo de competencias. Desde el siglo pasado se insiste en implementar modelos constructivistas centrados en el aprendizaje del alumno, pero hoy, se hacen indispensables.

Han surgido diferentes modalidades, metodologías y estrategias para innovar los procesos educativos, a partir de los ecosistemas académicos mediados por las tecnologías. Éstos se caracterizan actualmente por la presencia tecnológica en los planteles escolares, en el hogar y prácticamente en todos los ámbitos sociales. Se incrementan los dispositivos móviles que permiten el acceso a Internet y el estudiante tiene la oportunidad de emplear las tecnologías en diferentes escenarios.

Bates (2015), en su libro La enseñanza en la era digital examina los principios que en cierta forma guían la enseñanza eficaz en una época en la que todas las personas, y en particular los estudiantes, usan las tecnologías. Enfatiza la importancia del 
liderazgo en la toma de decisiones y también reconoce que cada instructor aplica formas únicas para enseñar, porque cada área temática es diferente.

Ante esta realidad, son inmensas las oportunidades que provee la tecnología a la educación, con una adecuada orientación, modelo y estrategia docente, que permita guiar al educando en el óptimo uso de ésta y facilitar la creación de entornos de aprendizajes conforme los principios constructivistas.

Esta teoría precisa que el conocimiento se construye a partir de acciones propias del individuo en pro de su aprendizaje. El papel del estudiante es activo y debe ser guiado en diferentes tareas significativas. Éstas a partir de la relación de los conocimientos previos y nuevos, de actividades experienciales y colaborativas que conducen a la formación de esquemas mentales.

Ortiz (2015) plantea que el conocimiento y su construcción tienen un componente idiosincrásico, al considerar el ritmo y forma de aprender, conocimientos previos, además de adecuar los materiales y contenidos a la realidad de los sujetos. De ahí la importancia de técnicas y diversidad de recursos; integrando técnicas como talleres, laboratorios, así como el pensamiento inductivo.

La tecnología a partir de sus características se convierte en herramienta mediática en pro del aprendizaje, donde docentes y estudiantes pueden interactuar, intercambiar, consultar y producir (Hernández, 2008). Por ejemplo, usar diferentes herramientas de la web 2.o como: redes sociales, wikis, blogs, entre otros, desde principios constructivistas, crean diferentes experiencias en el proceso de aprendizaje entre los estudiantes, vinculando de mejor forma lo que aprenden, y a su vez, expresarlo y demostrar los conocimientos nuevos adquiridos.

Lo anterior se relaciona con las tendencias y auge de modalidades educativas como el e-learning. En dicha modalidad las TIC se conforman como elementos esenciales para el desarrollo del proceso de enseñanza aprendizaje. Por tanto, en la actualidad se está apuntalando como una estrategia de formación continua a través de sus programas ya que representan flexibilidad y adaptabilidad con diseños pedagógicos abiertos para compartir contenidos, actividades, experiencias y procesos de autorregulación.

Como una extensión del e-learning se encuentran los Massive On-line Open Courses (MOOC's). En estos cursos se concibe la ubicuidad del aprendizaje, donde se trasforman las interacciones del docente y alumno, al trabajar colaborativamente, al poseer un conjunto de recursos que permiten el aprendizaje desde diferentes estilos y promover el acceso a la formación de diversos actores (Aguaded Gómez, 2013; Merado Del collado, 2016).

Vale destacar que estos cursos propician el desarrollo de habilidades en los estudiantes como el aprendizaje autónomo, el pensamiento crítico y la coevaluación entre pares, a partir de la metodología en la cual los productos también son valorados por los mismos estudiantes que participan en el curso.

La tendencia es que dichos cursos sean sostenibles y apuesten por una pedagogía basada en el multiculturalismo y la diversidad de contextos, sin tener necesariamente 
fines comerciales y favorecer el acceso a la formación de recursos, mediante la educación continua.

Otra modalidad educativa que cada vez toma mayor fuerza, a partir de la combinación de los ambientes virtuales y presenciales, es la b-learning. Según Turpo (2015) cuenta con una identidad propia al situarla como una estrategia innovadora y alternativa, de acuerdo con sus potencialidades en ambos ambientes, virtuales y presenciales, en los que se da indistintamente la integración y continuidad indistinguible de cada uno de sus espacios y componentes.

De acuerdo con González, Perdomo y Pascuas (2017); González (2018) el ecosistema de formación b-Learning requiere un arduo trabajo en la fase inicial (diseño instruccional) que proyecta el desarrollar habilidades cognitivas, competitivas, pensamiento crítico y constructivo para la resolución de problemas.

El éxito de la modalidad está en generar un ambiente donde confluyen varios componentes como: enfoques, recursos, medios tecnológicos y pedagógicos. Esta concepción se ve favorecida por los recursos que proporciona la web 2.0, que en torno a ésta componen una comunidad educativa en función de las dinámicas complejas entre los actores (autoridades, profesores, estudiantes, etc.) y otros elementos que convergen en dicha modalidad.

Tanto el b-Learning y el e-Learning son modalidades que han marcado la evolución de la educación presencial y a distancia al integrar de manera creativa la tecnología e incorporar el uso de los entornos personales y virtuales de aprendizaje. De acuerdo con Casquero (2013) la web 2.0 está teniendo impacto en dichas modalidades donde lo importante es la autoconfiguración y autogestión del entorno de aprendizaje por parte del alumno.

Otra forma de innovar es a partir de diferentes estrategias y herramientas como pueden ser: Animación en 3D, aplicaciones móviles educativas, repositorios digitales de objetos de aprendizaje y de e-books, la gamificación y videojuegos educativos, micromundos y mundos inmersivos, laboratorios y simuladores virtuales, entre otros. Estos recursos y ambientes no son exclusivos de una educación virtual, ya que pueden ser incorporados de forma innovadora en diferentes escenarios educativos. Éstos permiten que, en cualquier lugar, se generen entornos idóneos para el aprendizaje (Li, Qi, Wang y Wang, 2014).

Recientemente se ha extendido en planteles educativos la metodología didáctica denominada aula invertida (flipped classroom en inglés) como una opción para que el estudiante desarrolle habilidades de autoaprendizajes, mediante el uso de recursos tecnológicos. Es decir, el estudiante previamente realiza actividades que implican competencias de comprensión de la información, para luego en la clase presencial, se facilite la aplicación, análisis, síntesis y evaluación del conocimiento. Por lo que el docente se convierte en un facilitador que integra los aprendizajes, aclara las dudas, relaciona, compara y refuerza conceptos, para finalmente, realizar ejercicios prácticos, apoyándose en la metodología basada en problemas o estudio de casos, los cuales coadyuvan al aprendizaje significativo del estudiante. 
Para Berenguer (2016) fueron Jonathan Bergmann y Aaron Sams quienes consolidaron el término aula invertida o aula al revés. Son siete cuestiones básicas que Bergman y Sams (2014) recomiendan a los profesores para la aplicación del modelo de aula invertida:

- Introducir a los alumnos en el modelo. El docente define con exactitud las tareas del estudiante y fomenta el autoaprendizaje.

- Ver los videos e interactuar con ellos para identificar el contenido esencial.

- Formulación de preguntas interesantes. Esto permite comprobar si los alumnos han visto el video, revelar dudas y comprensión del tema explicado en el video.

- Preparar el ambiente de aprendizaje áulico. La disposición del mobiliario, el material debe permitir el desarrollo de las actividades y la cooperación.

- Gestión por parte del alumno de tiempos y cargas de trabajo. Al propiciar que aprenda a organizarse, detectar las prioridades y planificación del tiempo según sus necesidades.

- Cooperación entre estudiantes. Es importante que el estudiante aprenda a trabajar en colaboración con sus pares y organización de trabajo en equipo e individual.

- Construcción de un sistema de evaluación adecuado, mediante la diversificación de instrumentos de evaluación formativa.

Esta forma de aprendizaje proporciona una excelente plataforma para organizar actividades innovadoras en el aula, en las que se desarrollan las habilidades cognitivas de orden superior y el aprendizaje interdisciplinario principalmente en las áreas de las ingenierías, donde es requerido habilidades necesarias para la resolución de problemas (Li y Daher, 2016).

Actualmente existen investigaciones que pretenden comprobar la efectividad de la metodología en diferentes escenarios y niveles educativos. Tal es el caso de estudio realizado por Galindo Domínguez (2018) cuyos hallazgos principales son: Las investigaciones giran principalmente en el nivel superior, de tipo empíricos descriptivos, estudios de casos o cuasi experimentales. En donde más se ha aplicado, es en asignaturas como matemáticas, ciencias de la salud, derecho, entre otras. Estos se han realizado principalmente en EEUU, China, España y en América latina en Ecuador, México y Colombia.

Fortanet, Díaz, Pastor y Ramón (2013); Tourón y Santiago (2015); Berenguer (2016); Del Pino, et al. (2016), han demostrado los beneficios del aula invertida como son: Que el docente cuente con más tiempo en la clase presencial para atender las necesidades diferenciadas de los educandos. A su vez, el propio estudiante se involucra y se introduce en la materia de una forma mucho más participativa, interesada y activa.

Al mismo tiempo proporciona al alumno la posibilidad de repasar los contenidos y aprender a su ritmo con apoyo del profesor y en las clases se crea un ambiente de 
aprendizaje colaborativo. De este modo, existe una complementación entre la técnica del flipped classroom y el aprendizaje cooperativo: "las tareas -actividades-, también comúnmente conocidas como tareas, se realizan conjuntamente y en cooperación con el grupo ya que, el docente traslada el tiempo empleado a la explicación de la materia, al método flipped classroom o tarea en casa" (Fortanet, Díaz, Pastor y Ramón, 2013, p. 4).

Dicho enfoque está centrado en un aprendizaje activo del estudiante y no en que el docente sea el centro del proceso. Martín Rodríguez y Santiago Campión (2015) lo consideran un enfoque global y multidimensional, conlleva necesariamente un cambio metodológico en las aulas. Es decir, la incorporación en las aulas de metodologías activas, inductivas y colaborativas. Por lo tanto, se invierte la dinámica respecto a la metodología más tradicional en la que el profesor expone la materia en clase, y con posterioridad, el estudiante estudia el contenido, realiza ejercicios y tareas en casa como complemento a su estudio.

Existen algunos inconvenientes del modelo como lo señalan Madrid, Angulo, Prieto et al. (2018); Domínguez, Pepín, Moros y Villarraga (2017) quienes precisan que el éxito de esta metodología depende en gran medida del involucramiento del estudiante, al realizar las tareas de aprendizaje autónomo en casa, además de que demanda del profesor una adecuada integración de las habilidades prácticas y lo trabajado en casa; de tiempo para la planificación o selección de los contenidos que pondrá a disposición de los estudiantes. Por último, el acceso y uso de la tecnología en la cual se distribuyen los materiales.

La metodología del aula invertida es una estrategia educativa con mayor proyección a futuro, ya que se relaciona con los conceptos de docencia aprendida (lessons learned) y enseñanza semi-presencial (blended learning) que contribuye a desarrollar entre otras las competencias de autogestión, trabajo en equipo y habilidades informacionales de los alumnos.

El éxito de esta metodología depende de factores como: la calidad del material disponible en línea, la apropiada organización de las clases presenciales, donde el docente detecta las dificultades que conlleva el aprendizaje previo de los estudiantes y utiliza los recursos idóneos para corregir los errores de comprensión que se pueden producir y, en definitiva, guiar el aprendizaje de un grupo que puede o no ser muy numeroso y heterogéneo, a la apropiación y movilización de sus competencias tanto personales como profesionales, fomentando además la interacción estudianteprofesor y procurar una atención personalizada.

Estudios realizados en China por Chien-Yuan y Cheng-Huan (2018) han demostrado que los constructos del aprendizaje invertido y la generación de preguntas del estudiante, tienen un impacto positivo en la motivación, las actitudes y el compromiso de aprendizaje entre los estudiantes. En contraste, aunque el sistema de respuesta instantánea también tiene un impacto positivo en el compromiso de los estudiantes, no influye en la motivación o en las actitudes. 
Pero hay otros factores que también influyen en el rendimiento de los estudiantes durante el curso, como por ejemplo, su grado de aceptación hacia una metodología que exige una dedicación constante para poder participar y seguir con el aprovechamiento durante las clases.

Las características de las generaciones actuales han permitido la aceptación de dicho modelo. Arrieta y Montes (2011) describen a los estudiantes a partir de las siguientes características: reciben información rápidamente, prefieren imágenes a textos, privilegian las multitareas, trabajan en red, tienen una gran inclinación por los juegos, las recompensas y satisfacciones inmediatas.

Los estudiantes desarrollan mentes hiper-textuales, caracterizándose por tener la habilidad de leer imágenes visuales, desarrollar competencias espaciales y visuales, aprender por descubrimiento, realizar varias tareas y responder rápidamente en el tiempo.

Algunos se convierten en consumidores de recursos de Internet, de ahí la importancia de la alfabetización digital, para que los jóvenes hagan uso adecuado de la información. Pero existen otros que son creadores de contenidos, principalmente de videos y podcast, a los que han denominado yotubers.

Los resultados del estudio de Bonilla del Río, Mantecón y Acebo (2018) demostraron que los jóvenes de edades comprendidas entre 18 a 25 años, crean y comparten contenidos personales, sin embargo, no producen y comparten contenido académico o información educativa en las diversas plataformas de la web 2.o. A su vez, universitarios de mayor edad, tienden a producir mayor número de contenido académico, por lo que son prosumidores más activos. Dicho estudio demostró que conforme van avanzando en su vida académica, van adquiriendo más conciencia sobre la utilidad de los recursos digitales con fines académicos.

De ahí surge el interés por incluir en el ámbito educativo el término prosumidor (productor-consumidor) que no sólo se limita a consumir y colaborar porque produce, crea, comparte y publica. Según Sarsa (2014), es necesario integrar dichas competencias de creación, publicación y colaboración. Apoyar al alumno mediante las herramientas de la web 2.0 que genera una mezcla ideal entre producción y consumo, es un buen punto de partida para conseguir que los estudiantes se interesen (consumidores) y después se involucren en ser productores-publicadores de tareas educativas, es por eso que esta metodología propone nuevos horizontes.

Redecker, Ala-Mutka y Punie (2010) establecen cuatro tipos de usos relacionados con el aprendizaje entre los medios sociales como son: acceso a contenidos de aprendizaje, creación y publicación de contenidos propios, conexión entre estudiantes, expertos y profesores, y colaboración entre estudiantes y profesores.

Según Andreu y Martín (2014) el integrar el concepto de prosumidores en la docencia universitaria ofrece métodos de aprendizaje eficaces para que el alumno se enfrente a la adquisición del conocimiento, al mismo tiempo que Rodríguez y Escofet (2006) precisan que esta tendencia potencializa el papel activo del discente e incentiva la motivación, ya que éste tiene voz y adquiere protagonismo creativo en 
su propio aprendizaje; de ahí que se considera como una estrategia constructivista a partir de los principios de aprender haciendo Learning by doing. El docente debe crear entornos de aprendizaje basados en el aprendiz considerando: intereses, motivación, conocimientos previos y evaluación feedback.

De ahí el interés de integrar en esta investigación el modelo de aula invertida con el desarrollo de estrategias de estudiantes prosumidores de videos. Esto a partir de las características generales de las generaciones actuales y los nuevos modelos de integración de tecnología.

\section{METODOLOGÍA}

Este trabajo presenta un estudio de caso de carácter descriptivo, referido a la intervención y aplicación de la metodología del aula invertida en alumnos del segundo semestre de Ingeniería en Computación del Centro Universitario de los Altos, de la Universidad de Guadalajara. Es importante destacar, que previo a la implementación de esta metodología, se diagnosticó al grupo focal, encontrando en ellos, que sus principales canales de recepción y apropiación del aprendizaje eran los estilos visuales y kinestésicos.

Presentar el diseño, implementación y evaluación del modelo de aula invertida y actividad prosumidora, a partir de la integración de los trabajos creados por los estudiantes, contenidos en la segunda etapa de dicha metodología, es el principal objetivo y logro de este trabajo.

Esta experiencia educativa formó parte de la asignatura de "Programación", cuyo propósito principal fue implementar una estrategia, a partir de la creación y uso de recursos como videos, textos y casos prácticos, como se recomienda en la metodología del aula invertida. La asignatura consta de dos unidades de competencia y se diseñó un modelo docente en dos etapas. En la primera se aplicó la metodología tradicional del aula invertida, a partir de recursos seleccionados previamente por el docente. La segunda etapa consistió en que previamente los estudiantes realizaron un video (contenido: componentes visuales de NetBeans) a partir de la consulta de información y orientación del docente en sesiones fuera de clase.

Una vez que los estudiantes crearon, editaron y produjeron de forma colaborativa los videos, se publicaron en el canal de videos de Youtube. Posteriormente, los videos fueron revisados por el grupo previamente a cada sesión presencial. En total se realizaron 18 videos y en la sesión de clase se respondieron dudas y se consideraron casos prácticos. El modelo propuesto se puede observar más claramente en la figura 1.

Este modelo está sustentado en los principios constructivistas de aprender haciendo, el aprendizaje activo, el diseño de ambientes flexibles e interactivos de aprendizaje, donde el profesor es guía; por último, se despertó el interés y motivación del alumno en pro de su aprendizaje, fue importante considerar sus conocimientos previos y el respeto de los ritmos de aprendizaje. Esto genera que el estudiante dé 
significatividad a su aprendizaje a partir del desarrollo de habilidades prosumidoras y colaborativas.

Figura 1. Modelo de intervención de Flipped Teaching-prosumidor

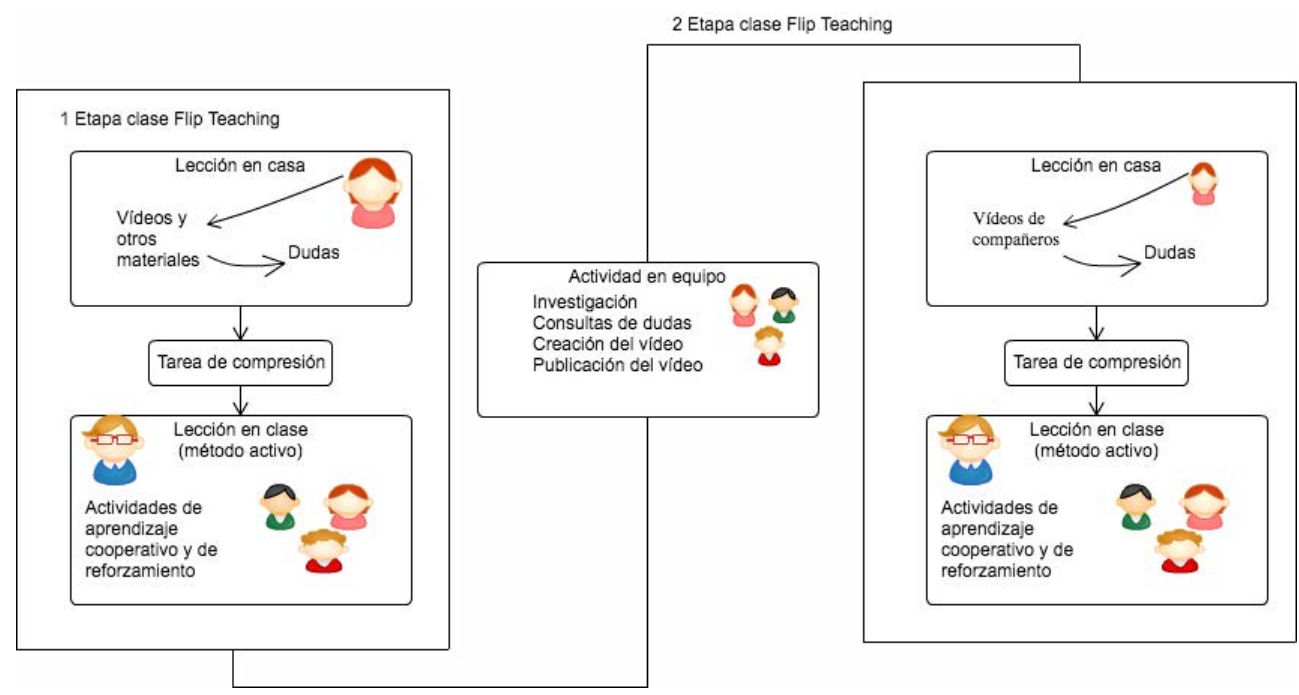

Después de implementar la estrategia, se evaluó mediante un estudio descriptivo que se realizó entrela población del conjunto completo de estudiantes del primer curso de "Programación" del segundo ciclo escolar (2018-A) de la carrera de Ingeniería en Computación $(\mathrm{N}=35)$. De acuerdo con la muestra censal no probabilística, dado el sesgo de autoselección por sujetos voluntarios. Aun así, se logró obtener una muestra de 24 alumnos que respondieron el cuestionario, lo que representa un muestreo representativo de un conjunto de 35 estudiantes, al considerar un nivel de confianza del 90\% y 10\% de margen de error.

En el instrumento para recoger la información, se utilizó el cuestionario tipo escala de Likert diseñado y adaptado a partir de la propuesta de García y Rodríguez (2016), que consideran las categorías de: percepción de la metodología respecto al aprendizaje; interacción docente-estudiante, estudiante-estudiante; ritmos de aprendizaje; colaboración; resolución de problemas y motivación. Además, se agregaron cinco preguntas adicionales para detectar la actitud del estudiante a la creación de contenido y el grado de aceptación del modelo y estrategia promotora de prosumidores.

Para medir el éxito de la estrategia se realizó un estudio longitudinal comparativo de muestras independientes, tomando como referencia el indicador obtenido en las evaluaciones del desempeño docente que se realiza de manera institucional, al término de cada ciclo escolar, a partir de un instrumento con 21 ítems divididos 
en las categorías de docencia y curso; así como la comparación del promedio de la calificación grupal del ciclo escolar 2017-A y 2018-A. Las condiciones de los grupos fueron igual en cuanto a: Asignatura, profesor, y semestre de impartición.

\section{RESULTADOS}

Los resultados del instrumento aplicado llevan a considerar el impacto favorable causado en los alumnos sujetos de este estudio, a partir de la metodología del aula invertida empleada como estrategia promotora de estudiantes prosumidores que, en trabajos análogos y futuras investigaciones se pueden comprobar y ampliar los resultados positivos de esta aplicación durante el ciclo escolar 2018-A (enero a mayo de 2018). Los hallazgos principales se muestran en la tabla 1.

Tabla 1. Resultados generales por ítem

\begin{tabular}{|c|c|c|c|c|c|c|c|c|c|c|}
\hline \multirow[t]{2}{*}{ Ítem } & \multicolumn{2}{|c|}{$\begin{array}{l}\text { Muy de } \\
\text { acuerdo }\end{array}$} & \multicolumn{2}{|c|}{$\begin{array}{c}\text { De } \\
\text { acuerdo }\end{array}$} & \multicolumn{2}{|c|}{$\begin{array}{c}\text { Ni de } \\
\text { acuerdo/ } \\
\text { ni en } \\
\text { desacuerdo }\end{array}$} & \multicolumn{2}{|c|}{$\begin{array}{l}\text { En desa- } \\
\text { cuerdo }\end{array}$} & \multicolumn{2}{|c|}{$\begin{array}{c}\text { Total- } \\
\text { mente en } \\
\text { desa- } \\
\text { cuerdo } \\
\end{array}$} \\
\hline & $\mathrm{F}$ & $\%$ & $\mathrm{~F}$ & $\%$ & $\mathrm{~F}$ & $\%$ & $\mathrm{~F}$ & $\%$ & $\mathrm{~F}$ & $\%$ \\
\hline $\begin{array}{l}\text { La actividad de ver videos } \\
\text { ayudaron a comprender el tema. }\end{array}$ & 7 & 29 & 14 & 58.4 & 1 & 4.2 & 0 & 0 & 2 & 8.4 \\
\hline $\begin{array}{l}\text { La combinación de las } \\
\text { actividades teóricas y prácticas } \\
\text { apoyan el aprendizaje. }\end{array}$ & 5 & 21 & 12 & 50 & 1 & 4 & 6 & 25 & 0 & o \\
\hline $\begin{array}{l}\text { Interacciones positivas con el } \\
\text { profesor. }\end{array}$ & 9 & 37 & 12 & 49.5 & 3 & 13 & 0 & 0 & 0 & o \\
\hline $\begin{array}{l}\text { Relaciones positivas entre tus } \\
\text { compañeros en la clase. }\end{array}$ & 14 & 58 & 7 & 29 & 3 & 13 & 0 & 0 & 0 & 0 \\
\hline $\begin{array}{l}\text { La metodología dispone mejor } \\
\text { acceso a los materiales y } \\
\text { contenidos. }\end{array}$ & 8 & 33.5 & 14 & 58.5 & 2 & 8 & 0 & 0 & 0 & 0 \\
\hline $\begin{array}{l}\text { Ver videos y realizar actividades } \\
\text { en clase permitió sentirte } \\
\text { motivado frente al aprendizaje. }\end{array}$ & 6 & 25 & 11 & 46 & 6 & 25 & 0 & o & 1 & 4 \\
\hline $\begin{array}{l}\text { Ver videos en casa; favorece } \\
\text { ritmos de aprendizaje y } \\
\text { compresión de conceptos. }\end{array}$ & 8 & 33 & 12 & 50 & 4 & 17 & 0 & 0 & 0 & o \\
\hline $\begin{array}{l}\text { La producción y compartir } \\
\text { el video permite mejorar los } \\
\text { aprendizajes y desarrollar } \\
\text { habilidades de comunicación. }\end{array}$ & 7 & 29 & 15 & 63 & 2 & 8 & o & 0 & 0 & o \\
\hline
\end{tabular}




\begin{tabular}{|c|c|c|c|c|c|c|c|c|c|c|}
\hline \multirow[t]{2}{*}{ Ítem } & \multicolumn{2}{|c|}{$\begin{array}{l}\text { Muy de } \\
\text { acuerdo }\end{array}$} & \multicolumn{2}{|c|}{$\begin{array}{c}\text { De } \\
\text { acuerdo }\end{array}$} & \multicolumn{2}{|c|}{$\begin{array}{c}\text { Ni de } \\
\text { acuerdo/ } \\
\text { ni en } \\
\text { desacuerdo }\end{array}$} & \multicolumn{2}{|c|}{$\begin{array}{l}\text { En desa- } \\
\text { cuerdo }\end{array}$} & \multicolumn{2}{|c|}{$\begin{array}{l}\text { Total- } \\
\text { mente en } \\
\text { desa- } \\
\text { cuerdo }\end{array}$} \\
\hline & $\mathrm{F}$ & $\%$ & $\mathrm{~F}$ & $\%$ & $\mathrm{~F}$ & $\%$ & $\mathrm{~F}$ & $\%$ & $\mathrm{~F}$ & $\%$ \\
\hline $\begin{array}{l}\text { El generar el video motivó el } \\
\text { aprendizaje sobre el tema. }\end{array}$ & 7 & 29 & 14 & 58 & 3 & 13 & 0 & 0 & 0 & 0 \\
\hline $\begin{array}{l}\text { Ver videos producidos por } \\
\text { los compañeros apoyó el } \\
\text { aprendizaje. }\end{array}$ & 10 & 41.5 & 10 & 41.5 & 3 & 13 & 1 & 4 & 0 & 0 \\
\hline $\begin{array}{l}\text { La metodología permitió la } \\
\text { participación, la resolución de } \\
\text { problemas y el pensamiento } \\
\text { crítico. }\end{array}$ & 5 & 21 & 14 & 58 & 4 & 17 & 1 & 4 & 0 & 0 \\
\hline $\begin{array}{l}\text { La metodología permitió, el } \\
\text { aprendizaje activo y experiencial }\end{array}$ & 9 & 37.5 & 9 & $37 \cdot 5$ & 5 & 21 & 1 & 4 & 0 & 0 \\
\hline $\begin{array}{l}\text { La metodología toma en cuenta } \\
\text { los puntos fuertes, debilidades e } \\
\text { intereses por parte del profesor. }\end{array}$ & 8 & 33 & 9 & 38 & 6 & 25 & 1 & 4 & 0 & 0 \\
\hline
\end{tabular}

Nota: F=Total de frecuencia por escala de Likert. Fuente: Elaboración propia.

Sobre la percepción de la metodología con respecto al aprendizaje, los resultados demuestran una tendencia positiva en la primera etapa de la metodología ya que el $29 \%$ de los alumnos manifiestan estar "muy de acuerdo" y un $58 \%$ "de acuerdo" en que las actividades de consultar videos ayudaron a la compresión del tema.

En una menor proporción considera que la combinación de actividades prácticas y teóricas están apoyando el aprendizaje, ya que el $21 \%$ respondió estar "muy de acuerdo" y el 50\% "de acuerdo".

Con relación a la segunda etapa del modelo "prosumidora de videos", la percepción del estudiantado fue mejor que la primera etapa ya que el $29 \%$ manifestó estar "muy de acuerdo" con esta estrategia y el $63 \%$ estar "de acuerdo", pues permite mejorar sus aprendizajes y desarrollar habilidades de comunicación.

Uno de los resultados más positivos de esta intervención se refiere a las interacciones docente-estudiante, estudiante-estudiante pues los resultados más altos corresponden a este rubro. En cuanto a las relaciones entre iguales, 58\% manifiesta estar "muy de acuerdo" y el 29\% estar "de acuerdo" en que la metodología implementada aumentó las relaciones positivas entre sus compañeros de clase.

De la relación docente-estudiante el 37.5\% manifiesta estar de "muy de acuerdo" y el 49.5\% "de acuerdo" en que se mejoraron las interrelaciones con el profesor. La comparativa entre ambos resultados se puede observar en la figura 2. 
Figura 2. Percepción de las interacciones en la metodología

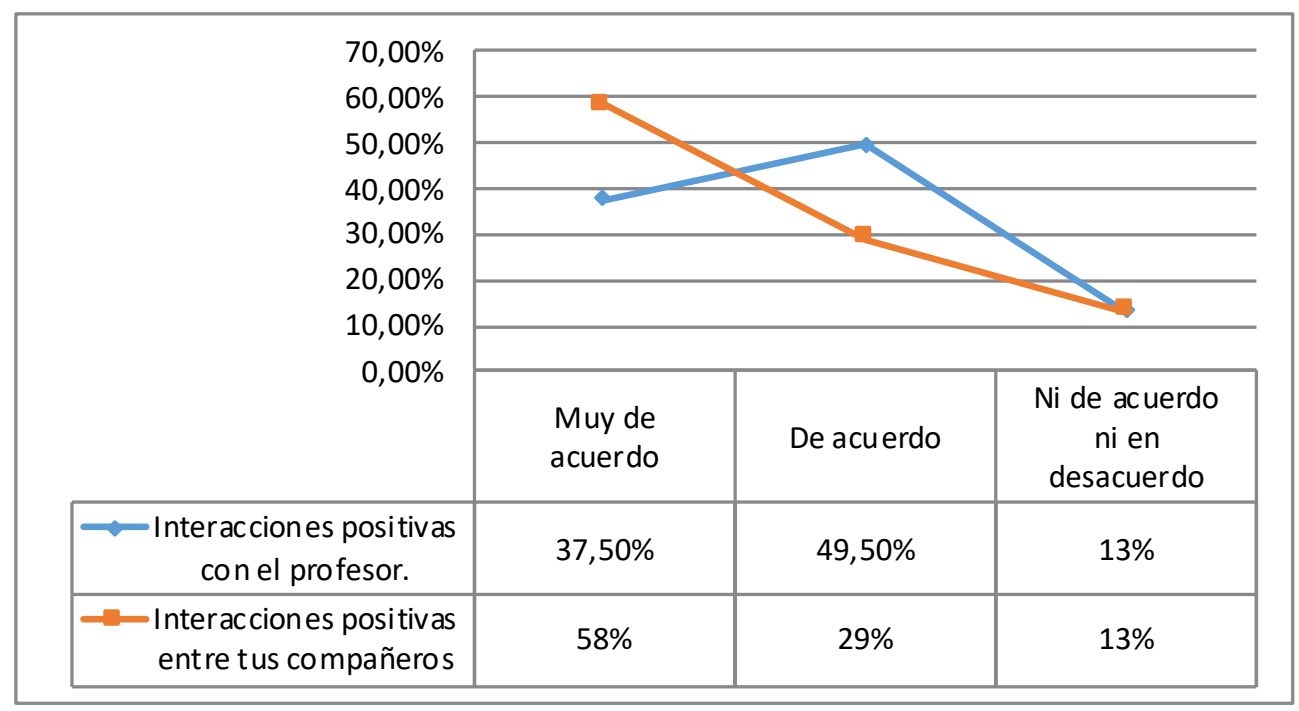

Fuente: Elaboración propia.

En la categoría de colaboración y difusión de videos, los resultados son positivos ya que el $41.6 \%$ manifiesta estar "muy de acuerdo" y también el $41.6 \%$ "de acuerdo" en que ver los videos de los compañeros favoreció el aprendizaje.

El desarrollo de habilidades para resolver problemas se considera que la metodología propuesta es buena, ya que el 21\% externó estar "muy de acuerdo” y el $58 \%$ "de acuerdo" con la experiencia propuesta.

A partir de la implementación de la metodología, la tendencia de su motivación fue muy semejante a la percepción en pro del aprendizaje, porque en la primera etapa el alumno se percibe menos motivado ya que el $25 \%$ respondió estar "muy de acuerdo" y el 46\% "de acuerdo" en que ver los videos y realizar actividades, los motivó para mejorar su aprendizaje.

El resultado más alto se observó en la segunda etapa, donde el alumno contestó que en un 29\% está "muy de acuerdo" y el 58\% manifiesta estar "de acuerdo" en que aumentó su motivación al momento de crear y ver el video de sus compañeros. Estos resultados comparativos se pueden observar claramente en la figura 3. 
Figura 3. Percepción respecto a la metodología por etapas en relación con la motivación

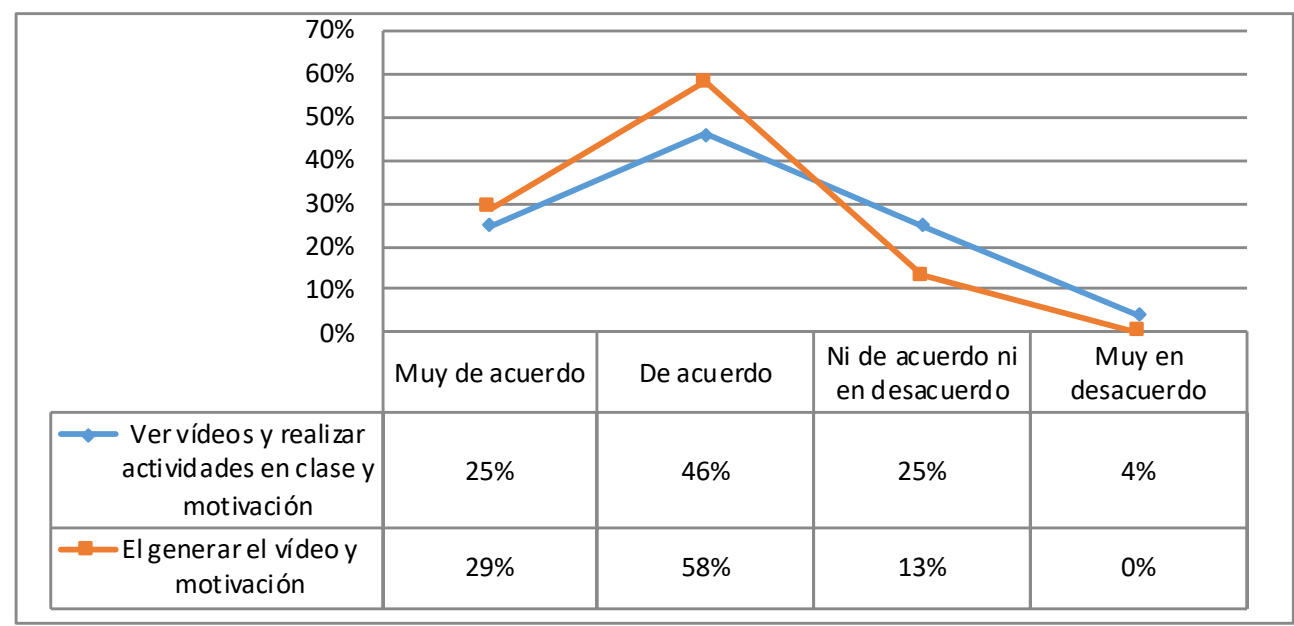

Fuente: Elaboración propia.

La figura 4 presenta resultados de los principios de aprendizaje activo y experiencial. Sólo el 4\% manifiesta estar en "desacuerdo", mientras que en conjunto estar de acuerdo suma el 75\%. El 21\% restante mostró una actitud neutral al respecto. Por último, los alumnos manifestaron estar "muy de acuerdo", 33\% y "de acuerdo", 50\% que la metodología ayudó a respetar los ritmos de aprendizaje y apoyó la comprensión de conceptos.

Figura 4. Percepción del modelo respecto al aprendizaje activo y experiencial

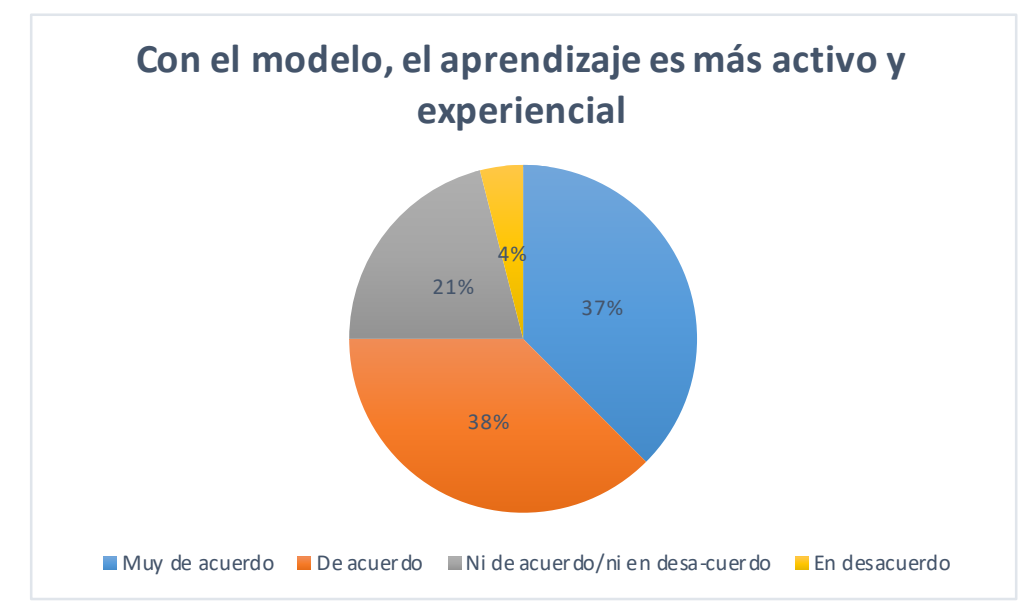

Fuente: Elaboración propia. 
Se consideró también una pregunta abierta de corte cualitativo, cuyos resultados indican que los alumnos consideran, en dicha experiencia, la importancia de dar continuidad al aprendizaje vivencial y práctico, porque generar videos fue aceptado por ellos; en oposición, sólo dos personas opinaron que los videos vistos en la primera fase eran mejores que los de sus compañeros.

Con la finalidad de comprobar la eficiencia de la metodología del modelo del aula invertida, se realizó un estudio comparativo para tener una vista longitudinal de los resultados académicos en cuanto al desempeño del alumno y del docente del grupo 2017-A y el mismo grupo durante el ciclo 2018-A en el que se aplicó la experiencia.

Su calificación en puntos aumentó un $5.5 \%$ el promedio grupal del desempeño del alumno con respecto al grupo anterior donde no se aplicó dicha metodología; además, se observa un incremento del $2.7 \%$ en el promedio de la evaluación del curso en la valoración que realizaron los estudiantes sobre el profesor en el curso. Estos resultados se muestran en la tabla 2.

Tabla 2. Comparativa entre el grupo anterior y el grupo que llevó el modelo

\begin{tabular}{|l|l|l|}
\hline \multicolumn{3}{|c|}{ Asignatura : Programación } \\
\hline \multicolumn{1}{|c|}{ Aspecto a comparar } & Grupo 2017-A & Grupo 2018-A \\
\hline Promedio de evaluación de la docencia & 9,17 & 9,2 \\
\hline Promedio de evaluación del curso & 8,78 & 9,05 \\
\hline Promedio de evaluación general del desempeño & 8,98 & 9,13 \\
\hline Promedio del desempeño del alumno en puntos & 81,78 & 87,37 \\
\hline
\end{tabular}

\section{DISCUSIÓN Y CONCLUSIONES}

Conforme los resultados, se puede ver que a partir de las características de la población atendida, la implementación de dicho modelo incidió positivamente en el aprendizaje del estudiante, según la premisa implícita del aula invertida donde se precisa que el alumno con antelación a la clase presencial, debe haber estudiado los contenidos de la unidad de aprendizaje, mediante materiales disponibles en línea, especialmente videos. Se pudo comprobar un incremento en cuanto a los valores de la evaluación general del desempeño y el promedio grupal con respecto al curso anterior en el que se implementó diferente metodología.

Trabajar con videos en casa, permite al estudiante ajustarse a su propio ritmo para comprender los conceptos. Al mismo tiempo que la metodología permite a los alumnos mayores posibilidades de participar en la resolución de problemas y desarrollar su pensamiento crítico.

Se observa una percepción positiva respecto al uso de los videos en casa como apoyo a su aprendizaje. Aunque se obtuvieron valores altos en la percepción de la 
integración de conocimientos teóricos de los videos, con los prácticos de las sesiones en clase, la percepción fue mejor respecto a la creación del video.

Por ello, se infiere que no todos los estudiantes percibieron una adecuada relación entre los conocimientos teóricos y prácticos, lo que hace necesario que, en próximas estrategias, se generen actividades que den continuidad a las actividades, tanto en casa como en la clase.

De todo lo anterior, se deduce que en la primera parte de la metodología es importante la promoción del trabajo individualizado y el pensamiento crítico para que el estudiante genere y registre las dudas a discutir durante las clases presenciales.

Estos resultados, concuerdan con los de McLean et al. (2016) donde demuestran que los estudiantes valoraran positivamente la interactividad, la creatividad y el compromiso con las tareas en pro del aprendizaje profundo, así como estar de acuerdo en que favorece su pensamiento crítico y activo.

Fueron favorables los resultados en torno a una actitud prosumidora, ya que los estudiantes se sienten motivados en la creación de contenidos para socializarlos con sus compañeros. Este aspecto es positivo, de acuerdo con Berenguer (2016) mejora el ambiente en el aula y la convierte en un espacio donde se comparten ideas, se plantean interrogantes y se resuelven dudas, fortaleciendo también el trabajo colaborativo y promoviendo una mayor interacción alumno-profesor.

En general, se demostró alta motivación y aceptación del modelo, compatibles a los resultados de Sarsa (2014), la integración de una estrategia prosumidora como parte del modelo, fue aceptada y valorada positivamente; esto refuerza lo que ya se ha mencionado, la necesidad de que las instituciones de educación superior fomenten políticas educativas para la incorporación de herramientas web 2.o donde el docente motive a sus estudiantes a explorar diferentes herramientas con una intencionalidad educativa, además de que el mismo docente sea mediador en la creación de hábitos de consumo y participación en la producción de contenidos educativos.

Este estudio sin intención de comparar las dos etapas del modelo destaca que en la primera el estudiante es menos activo y el docente es el prosumidor; mientras que en la segunda el alumno intercambia este papel, lo que permitió que la metodología propuesta fuera un detonante que influyó en la motivación del estudiante y permitió la colaboración y aprender haciendo. De acuerdo con Rodríguez y Escofet (2006) los estudiantes se posicionan de manera activa en la producción de contenidos, y en el hecho de que, para poder desarrollarlos, deben usar distintas herramientas basadas en las tecnologías de la información y de la comunicación.

El diseño pedagógico de esta intervención pudo demostrar el sustento constructivista desde la percepción del estudiante, porque al colocarlo como productor de sus conocimientos y de contenidos, asumió un rol activo, al consultar, resolver y producir productos educativos.

Cabe considerar que tal vez las percepciones de desacuerdo de la metodología sean causa de la gran influencia de concepciones tradicionales del aprendizaje, en las que al estudiante se le proporciona el conocimiento y es pasivo al momento de 
ser receptivo de lo que se pretende que aprenda, por lo que requiere mayor esfuerzo por parte del estudiante.

En sus estudios McLean et al. (2016) y Li y Daher (2016) comprueban que en este tipo de metodología, se pueden tener resultados negativos ya que los discentes menos comprometidos e involucrados opinan que existe mayor carga de trabajo que las clases tradicionales. En consecuencia, se da cuenta que tiene que adoptar un enfoque diferente frente al aprendizaje, al adquirir habilidades de trabajo independiente y administración del tiempo.

Para aplicaciones futuras, sería bueno examinar y rediseñar en clase las actividades, para que más estudiantes participen activamente en las discusiones. Esta metodología favorece la construcción del aprendizaje, siempre y cuando exista un compromiso activo de alumnos y docentes. Los primeros son capaces de un aprendizaje autorregulado, al participar de manera activa. El docente debe involucrarse en todo el proceso, desde la etapa de planificación y creación de contenidos, hasta el momento de guiar y evaluación de los aprendizajes.

Un modelo de integración del aula invertida y un enfoque prosumidor permite generar una unión metodológica útil para el desarrollo de la creatividad, la aclaración y apropiación de conceptos, al permitir un aprendizaje activo y profundo. El modelo instruccional, no consiste en un cambio de tecnología, sino en diseñar ambientes de aprendizajes. La relación entre la tecnología y la construcción del aprendizaje no es un hecho deliberado, debe existir un cambio de concepción del aprendizaje por parte del docente y del estudiante que derive en una trasformación metodológica, para integrar diversas estrategias, propias de la posición constructivista, como son la resolución de problemas, casos, el aprendizaje colaborativo y situado, entre otros.

De acuerdo con Bates (2015) lo sustancial desde la perspectiva del aprendizaje no es la elección de la tecnología sino la eficiencia, la experiencia en la elección y la correcta aplicación del método de enseñanza. Por lo que, en la medida que se generen diversas combinaciones de métodos y tecnologías; cambios en buenas prácticas docentes a partir de modelos adecuados al estilo de aprendizaje del aprendiz, es lo que va a hacer la diferencia en el desarrollo de competencias.

Como sugerencia al inconveniente de falta de compromiso de los estudiantes para ver los videos, es hacer consciente al estudiante de su papel activo como, por ejemplo: actividades previas a la clase que permitan recapitular y generar un blog de dudas respecto a las temáticas. Así al promover aprendizaje efectivo como lo dicen Roschelle et al. (2000) cuando están presentes las características de: compromiso activo, participación en grupo, interacción frecuente, y retroalimentación y conexiones con el contexto del mundo real.

Una limitación de este estudio es que no se pueden generalizar los resultados, porque sólo es una experiencia educativa. Es necesario replicar el estudio en más grupos y con muestras mayores, para correlacionar las percepciones de los estudiantes y el rendimiento académico demostrado en los cursos. Además, otra de las líneas de investigación es indagar las habilidades necesarias y requeridas para que 
los estudiantes puedan mejorar sus competencias como productores-consumidores al utilizar herramientas que provee la web 2.o.

\section{REFERENCIAS}

Aguaded Gómez, J. I. (2013). La revolución MOOCs, ¿una nueva educación desde el paradigma tecnológico? /The MOOC revolution: ¿A new form of education from the technological paradigm? Revista Comunicar, 21(41), 7-8. doi: 10.3916/C412013-a1.

Andreu Sánchez, C., y Martín Pascual, M. A. (2014). La educación audiovisual y la creación de prosumidores mediáticos. Estudio de caso. Revista Científica de Estrategias, Tendencias e Innovación en comunicación, (7), 131-147. doi: http:// dx.doi.org/10.6035/2174-0992.2014.7.8

Arrieta, A. C., y Montes, D. V. (2011). Alfabetización digital: uso de las tic's más allá de una formación instrumental y una buena infraestructura. Revista Colombiana de Ciencia Animal-RECIA, 3(1), 180-197.

Bates, A. W. (2015). Teaching in a digital age. Guidelines for desingning teaching and learning, 6. Recuperado de https://irl.umsl.edu/cgi/viewcontent. cgi?article $=1005$ \&context $=$ oer

Berenguer Albaladejo, C. (2016). Acerca de la utilidad del aula invertida o flipped classroom. En M. T. Tortosa Ybáñez, S. Grau Company y S. D. Álvarez Teruel (Coord.), XIV Jornadas de redes de investigación en docencia universitaria (1466 -1480). Alicante: Universidad de Alicante.

Bergmann, J., y Sams, A. (2014). Dale la vuelta a tu clase. Madrid: Ediciones SM.

Bonilla del Río, M., Diego Mantecón, J. M., y Lena Acebo, F. J. (2018). Estudiantes Universitarios: prosumidores de recursos digitales y mediáticos en la era de internet. Revista Aula Abierta, 47(3), 319-326.
Casquero (2013). Composición y estructura de redes personales en entornos de aprendizaje personales. En L. Castañeda y J. Adell (Eds.), Entornos Personales de Aprendizaje: claves para el ecosistema educativo en red. (151-160). Alcoy: Marfil.

Chien-Yuan y Cheng-Huan. (2018). Investigating the effects of flipped learning, student question generation, and instant response technologies on students' learning motivation, attitudes, and engagement: A structural equation modeling. Eurasia Journal of Mathematics, Science and Technology Educatios, 14(6), 2453-2466. doi: 14. 2453-2466. 10.29333/ejmste/89938.

Del Pino, B., Campos, B. P., Espinosa, A. P., y García, F. M. I. (2016). Utilización de la metodología de aula invertida en una asignatura de Fundamentos de Informática. Enseñanza y aprendizaje de ingeniería de computadores: Revista de Experiencias Docentes en Ingeniería de Computadores, (6), 67-75.

Domínguez, L. C., Sierra, D., Pepín, J. J., Moros, G., y Villarraga, A. (2017). Efecto del Aula Invertida Extendida a simulación clínica para la resucitación del paciente traumatizado: estudio piloto de las percepciones estudiantiles sobre el aprendizaje. Revista Colombiana de Anestesiología, (45), 4-11.

Fortanet, C., Díaz, C. G., Pastor, E. M., y Ramón, J. L. (2013). Aprendizaje cooperativo y flipped classroom. Ensayos y resultados de la metodología docente. En XI Jornadas de Redes de Investigación en Docencia Universitaria: Retos de futuro en la enseñanza superior: Docencia e investigación para alcanzar la excelencia 
académica (1151-1162). Instituto de Ciencias de la Educación.

Galindo Domínguez, H. (2018). Un metaanálisis de la metodología Flipped Classroom en el aula de Educación Primaria. Edutec. Revista Electrónica de Tecnología Educativa, (63), 73-85. doi: /10.21556/edutec.2018.63.983.

García, R., y Rodríguez, M. (2016). El aula invertida (flipped classroom) en Educación Primaria. Technical Report (PDF Available). Recuperado de https://www.researchgate.net/ publication/305474488 El aula invertida flipped classroom en Educacion Primar

González Aldana, M. A., Perdomo, K. V., y Pascuas, Y. (2017) Aplicación de las TIC en modelos educativos blended learning: una revisión sistemática de literatura. Sophia, 13(1), 144-154. doi: http://dx.doi. org/10.18634/sophiaj.13v.1i.36

González Fernández, M. O. (2018). Percepción del desempeño docenteestudiante en la modalidad mixta desde una mirada ecosistémica/Perception of teacher-student performance in the mixed mode from an ecosystemic perspective. RIDE Revista Iberoamericana para la Investigación y el Desarrollo Educativo, 8(16), 370-393. doi: 10.23913/ ridev8i16.346.

Hernández, S. (2008). El modelo constructivista con las nuevas tecnologías: aplicado en el proceso de aprendizaje. RUSC. Universities and Knowledge Society Journal, 5(2), 26-35.

Li, F. QI., J., Wang, G., y Wang, X. (2014), Traditional classroom vs e-learning in higher education: Difference between students' behavioural engagement. International Journal of Emerging Technologies in Learning, 9(2), 48-51.

Li, Y., y Daher, T. (2016). Integrating innovative classroom activities with flipped teaching in a water resources engineering class. Journal of Professional
Issues in Engineering Education and Practice, 143(1), 1-6. doi: 10.1061/(ASCE) EI.1943-5541.0000297.

Madrid García, E. M., Ángulo Armenta, J., Prieto Méndez, M. E., Fernández Nistal, M. T., y Olivares Carmona, K. M. (2018). Implementación de aula invertida en un curso propedéutico de habilidad matemática en bachillerato. Revista Apertura, 10(1), 24-39. doi: 10.18381/ Apv10n1.1149.

Martín Rodríguez, D., y Santiago Campión, R. (2015). ¿Es el flipped classroom un modelo pedagógico eficaz? Un estudio sobre la percepción de estudiantes de Primaria, ESO y Bachillerato. Revista Comunicación y Pedagogía, 285-286.

McLean, S., Attardi, S. M., Faden, L., y Goldszmidt, M. (2016). Flipped classrooms and student learning: not just surface gains. Advances in physiology education, 4O(1), 47-55. doi: 10.1152/ advan.00098.2015

Ortiz, D. (2015). El constructivismo como teoría y método de enseñanza. Revista Sophia, Colección de Filosofía de la Educación, 1(19), 93-110.

Mercado del collado, R. (2016). Cursos masivos abiertos en línea: oportunidad o amenaza. Universidades, (70), 53-68.

Redecker, C., Ala-Mutka, K., y Punie, Y. (2010). Learning 2.0 - The Impact of Social Media on Learning in Europe. JRC Technical Notes. Luxembourg. European Communities. Recuperado de http://ftp. jirc.es/EURdoc/JRC56958.pdf

Roschelle, J. et al. (2000). Changing how and what children learn in school with computer-based technologies. The Future of Children, 10(2), 76-10.

Rodríguez, J. L., y Escofet, A. (2006). Aproximación centrada en el estudiante como productor de contenidos digitales en cursos híbridos. RUSC. Universities and Knowledge Society Journal, 3(2), 20-28. 
Sarsa, J. (2014). El perfil prosumidor de los estudiantes en la web 2.0. Journal for Educators, Teachers and Trainers, 5(2), 74-87.

Tourón, J., y Santiago, R. (2015). El modelo Flipped Learning y el desarrollo del talento en la escuela: Flilpped Learning model and the development of talent at school. Madrid: Ministerio de Educación.

Turpo Gebera, O. (2015). Perspectiva de la convergencia pedagógica y tecnológica en la modalidad blended learning. Revista de Educación a Distancia, (39). Recuperado de https://revistas.um.es/red/article/ view/234261

\section{PERFIL ACADÉMICO Y PROFESIONAL DE LOS AUTORES}

María Obdulia González Fernández. Doctora en Sistemas y ambientes educativos por la Universidad de Guadalajara. Profesora investigadora del Departamento de Ingenierías del Centro Universitario de los Altos de la Universidad de Guadalajara además de participar como asesora invitada en la Maestría de Educación Básica de la Universidad Pedagógica Nacional de México. Responsable del cuerpo académico de gestión e innovación educativa y tecnología de la misma universidad. Su línea de investigación son los modelos y escenarios de integración de la tecnología en pro del aprendizaje.

E-mail: ogonzalez@cualtos.udg.mx

Pablo Huerta Gaytán. Cuenta con una maestría en Educación por la Universidad Autónoma de Guadalajara y es licenciado en Periodismo por la Escuela de Periodismo Carlos Septién García de la ciudad de México. Profesor adscrito al Departamento de Estudios Jurídicos, Sociales y de la Cultura, del Centro Universitario de los Altos. Integrante del cuerpo académico de gestión e innovación educativa y tecnología de la misma universidad. Se ha desempeñado en la investigación aplicada para impulsar tanto el periodismo público y divulgación del conocimiento, como la difusión cultural en la región Altos Sur de Jalisco, México. La educación y tecnología es su línea de investigación actualmente.

E-mail:phuertagaytan@gmail.com

Dirección:

Centro Universitario de los Altos

Universidad de Guadalajara

Av. Rafael Casillas Aceves No. 1200

Tepatitlán de Morelos, Jalisco (México)

Fecha de recepción del artículo: 16/11/2018

Fecha de aceptación del artículo: 15/01/2019

Fecha de aprobación para maquetación: 18/03/2019 